\title{
Unsteady Analyses of Valve Systems in Rocket Engine Testing Environments
}

\author{
Jeremy Shipman*, Ashvin Hosangadi ${ }^{\dagger}$, and Vineet Ahuja ${ }^{\ddagger}$ \\ Combustion Research and Flow Technology, Inc. (CRAFT Tech) \\ 6210 Keller's Church Road, Pipersville, PA 18947 \\ Phone: 215-766-1520/ Fax: 215-766-1524
}

\begin{abstract}
This paper discusses simulation technology used to support the testing of rocket propulsion systems by performing high fidelity analyses of feed system components. A generalized multi-element framework has been used to perform simulations of control valve systems. This framework provides the flexibility to resolve the structural and functional complexities typically associated with valve-based high pressure feed systems that are difficult to deal with using traditional Computational Fluid Dynamics (CFD) methods. In order to validate this framework for control valve systems, results are presented for simulations of a cryogenic control valve at various plug settings and compared to both experimental data and simulation results obtained at NASA Stennis Space Center. A detailed unsteady analysis has also been performed for a pressure regulator type control valve used to support rocket engine and component testing at Stennis Space Center. The transient simulation captures the onset of a modal instability that has been observed in the operation of the valve. A discussion of the flow physics responsible for the instability and a prediction of the dominant modes associated with the fluctuations is presented.
\end{abstract}

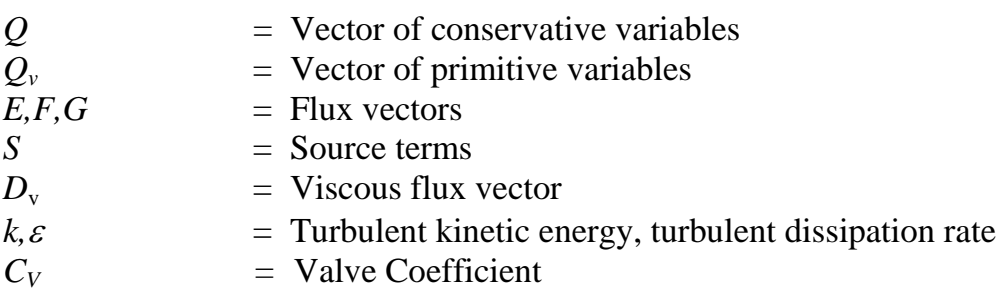

\section{Introduction}

$\mathrm{T}$

HE ability to accurately and efficiently model feed system elements such as control valves, pressure regulators, and other flow control and metering devices is necessary to support liquid rocket propulsion system test programs. Simulation technology supporting such programs must meet the dual requirements of working accurately in complex flow regimes and having efficient solution turn-around times that are suitable to support real-time testing. Such analyses are particularly important for the reliable operation of the high pressure valves found on test stands used for full rocket engine test firing and/or the testing of components such as cryogenic pumps. A number of flow characteristics important to the valve performance, including sensitivity to thermal conditions for cryogenic fluids, multiphase flow regimes, and transient anomalies stemming from hydrodynamic instabilities, valve timing, and system-based dynamic instabilities, are difficult to identify with empirical correlations and low-dimensional analysis methods. The use of such methods is restricted to the analysis of global performance characteristics. It is therefore necessary for higher fidelity simulation tools to be developed for valve-based systems.

The analysis of control valve type systems using high fidelity Navier-Stokes based methods is typically very challenging due to the structural and functional complexity associated with such systems ${ }^{1}$. Structurally, a valve

\footnotetext{
* Research Scientist, AIAA Member.

${ }^{\dagger}$ Principal Scientist, AIAA Senior Member.

${ }^{\ddagger}$ Research Scientist, AIAA Senior Member.
} 
system can consist of a complex network of embedded plugs, diaphragms, manifolds, and arterial branches with complicated junctions. For example, a control valve such as the pressure regulator valve illustrated in Figure 1 can have a flowpath that consists of multiple channels feeding a complex-shaped chamber that contains a variable area poppet with tight clearances in the seat region. The flow enters and exits the valve through complex-shaped pipe junctions and valve body. Traditional CFD tools have severe limitations imposed on them, both in terms of accuracy and efficiency, while carrying out high fidelity analyses of such complicated systems. These limitations stem from the topological constraints imposed by the complexity of the geometry and the diversity of spatial and temporal scales that must be locally resolved in the flow domain. Functionally, the characterization of such valve-based feed systems can be difficult due to a number of performance related transient issues such as fluidic/hydrodynamic instabilities arising from secondary flows, recirculating regions, and shedding phenomena that are caused by the complexities in the flowpath geometry. Such instabilities are usually not localized, and can couple with the valve motion and structural elements leading to undesirable system-wide fluctuations at high flow conditions.

The effective support of rocket engine and component testing must therefore be provided by simulation technology that has matured to include the following attributes: (1) the capability for analysis of diverse flow regimes including highly compressible, low Mach number, reacting flows, and cryogenic environments, (2) submodels for the accurate representation of the heat and mass transfer mechanisms accounting for phase change processes such as cavitation, (3) flexible and rapid grid generation of a network of complex shapes, (4) the ability to control local grid resolution in order to resolve important features of the flow physics, and (5) the high fidelity analysis of transient phenomena in order to accurately identify the dominant frequencies affecting performance and valve timing issues. While previous work by the authors has focused on the first two items ${ }^{2,3}$, the studies presented here are concerned with the final three. In this paper, we demonstrate the simulation capability of a multi-element unstructured numerical framework, CRUNCH CFD ${ }^{\circledR}$, for complex valve applications. This simulation capability has been proven to provide efficient and accurate solutions for complex turbomachinery applications in the past ${ }^{4}$. In this paper, we discuss both steady and unsteady simulations that were performed using a multi-element grid approach (hexahedral, tetrahedral, prismatic and pyramidal elements), providing a cell topology that is tailored to the structural complexities of the valve flowpath, and resulting in a well resolved, high quality grid. The numerical framework of the code is detailed in section II. In section III, a series of steady state, axisymmetric simulations using CRUNCH CFD ${ }^{\circledR}$ is presented for a cryogenic control valve at different plug settings. The flow coefficient as a function of plug setting is compared to both experimental data and simulation results obtained at Stennis Space Center. In section IV, detailed steady and unsteady analyses are presented for the valve geometry illustrated in Figure 1, representing a pressure regulator valve found in the high pressure feed systems of the rocket test facilities at NASA Stennis Space Center. Results from the steady simulations reveal a rich variety of flow phenomena over a multitude of length scales. The transient analysis was performed with a fixed plug setting and captures a dominant instability with a 4 $\mathrm{KHz}$ frequency. In addition to the simulation results, the multi-element

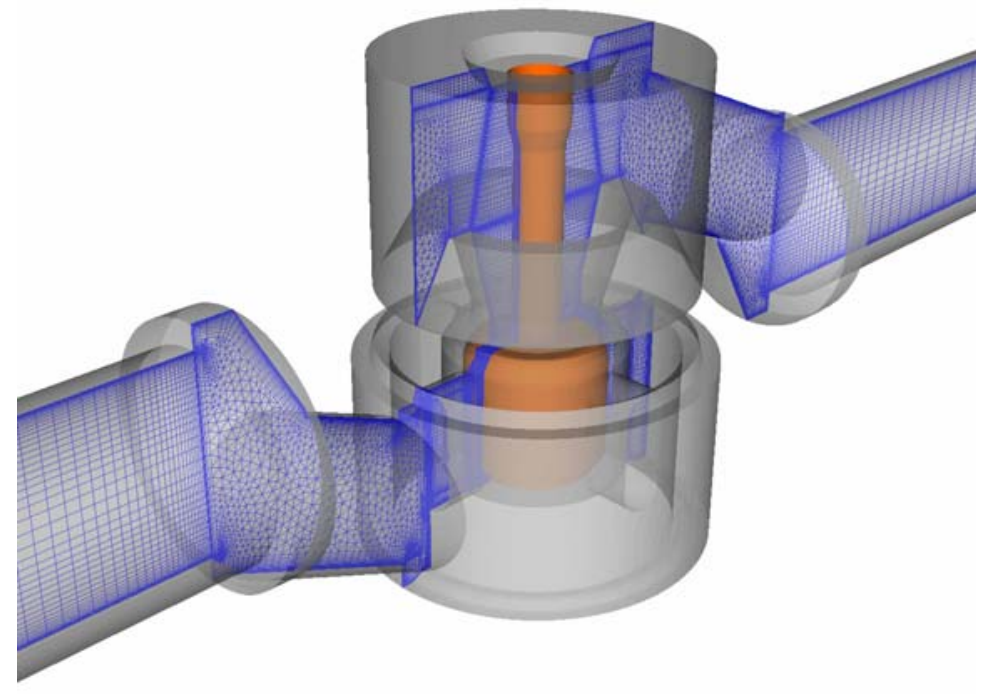

Figure 1. Pressure regulator valve geometry with the multi-element grid (shown along the symmetry plane) used to mesh the valve flowpath. strategy used in the grid construction is presented in this section.

\section{Crunch CFD ${ }^{\circledR}$ Computational Framework}

The CRUNCH CFD ${ }^{\circledR}$ code has a hybrid, multi-element unstructured framework that allows for a combination of tetrahedral, prismatic, and hexahedral cells ${ }^{5}$. The grid connectivity is stored as an edge-based, cell-vertex data structure, where a dual volume is obtained for each vertex by defining surfaces that cut across edges coming to a 
node. An edge-based framework is attractive for dealing with multiple element types, since the dual surface areas for each edge can include contributions from different element types making the inviscid flux calculation "grid transparent."

The integral form of the conservation equations are written for a dual control volume around each node as follows:

$$
\Gamma_{p} \frac{\Delta Q_{v} V}{\Delta t}+\int_{\alpha \Omega_{i}} F\left(Q_{v}, n\right) d s=\int_{\Omega_{i}} S d V+\int_{\Omega_{i}} D\left(Q_{v}, n\right) d s
$$

The inviscid flux procedure involves looping over the edge list and computing the flux at the dual face area bisecting each edge. The Riemann problem at the face is then solved using higher order reconstructed values of primitive variables at the dual face. A second-order linear reconstruction procedure following Ref. 6 is employed to obtain a higher-order scheme. For flows with strong gradients, the reconstructed variables are limited to obtain a stable TVD scheme. While the inviscid flux as outlined above is grid-transparent, the viscous flux calculation is computed on an element basis because the diffusion of scalars is sensitive to the skewness of the element type.

For the efficient computation of large 3D problems, a parallel framework for distributed memory systems has been implemented, along with a time-marching implicit solution procedure. The sparse implicit matrix is derived using an Euler explicit linearization of the first-order flux, and a variety of iterative sparse matrix solvers, e.g. GMRES, Gauss-Seidel procedure, are available in the code ${ }^{5,7}$. The parallel framework is implemented by partitioning the grid into sub-domains, with each sub-domain residing on an independent processor. The message passing between processors has been implemented using the standard Message Passing Interface (MPI) libraries to provide portability across platforms. The CRUNCH CFD ${ }^{\circledR}$ code has an integrated grid refinement and coarsening package operational within a multi-element framework ${ }^{8}$.

\section{Cryogenic Control Valve}

A series of simulations was performed for a cryogenic control valve. In these simulations, the multi-element CRUNCH CFD ${ }^{\circledR}$ framework was used to estimate the valve flow coefficient as a function of the plug setting. The aim of this exercise was to compare the valve flow coefficient curve to experimental data and simulations performed at Stennis Space Center ${ }^{9}$. The valve configuration and the grid that was used for the simulations are shown in Figure $2 \mathrm{a}$ and $2 \mathrm{~b}$, respectively. The plug/seat portion of the geometry exactly matches the configuration of the actual valve.
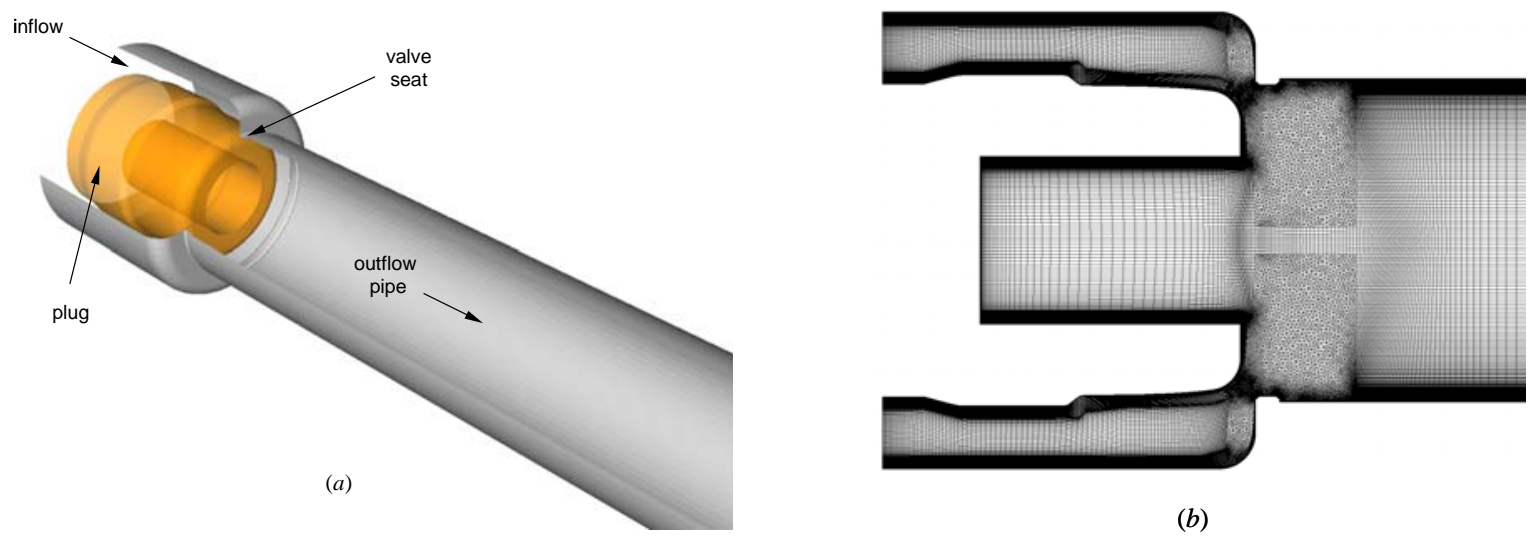

(b)

Figure 2. Illustration of (a) geometric configuration of the cryogenic control valve, and (b) detail of the symmetry plane of the axisymmetric grid (mirrored about the axis for clarity).

However, in the simulations the entire valve geometry was approximated to be axisymmetric. For the purpose of simulating the cryogenic control valve, this is a valid approximation because the discharge and inlet ducts are located away from the plug/seat region and there are little three-dimensional flow distortions affecting this region. A series of simulations was performed on this valve configuration corresponding to five different valve settings ranging from $45 \%$ to $75 \%$ open. Liquid nitrogen was considered the working fluid in the control valve for all the simulations with operating conditions at $162 \mathrm{R}$ and 3000 psi. The density of liquid nitrogen under the operating conditions was $800 \mathrm{~kg} / \mathrm{m} 3$ and the valve operated with a mass flow rate that ranged from 100-300 lbs/sec. Since the operating conditions of the valve are far from the vapor pressure and variations in pressure across the valve seat are 
small, there is minimal phase change of the fluid and density variations are negligible. Therefore, for the sake of the simulations, the temperature was assumed to be constant in the control valve and the liquid nitrogen was modeled as an incompressible liquid with constant density and molecular viscosity. This assumption was imposed to get a backto-back comparison with the simulations performed at Stennis Space Center with identical operating conditions.

A series of five simulations was performed with valve settings at $45 \%, 50 \%, 65 \%, 70 \%$ and $75 \%$ open. The mass flow rates utilized for these simulations were $100 \mathrm{lbm} / \mathrm{sec}, 150 \mathrm{lbm} / \mathrm{sec}, 150$ $\mathrm{lbm} / \mathrm{sec}, \quad 300 \mathrm{lbm} / \mathrm{sec}$, and $300 \mathrm{lbm} / \mathrm{sec}$ respectively. The simulations were run on a parallel PC cluster (16 processors) to provide rapid turnaround times consistent with those required to support testing (5-6 hours). The numerical simulations were performed using the formulation described in the previous section with a wall function procedure in the vicinity of viscous boundaries specified in conjunction with the high-Reynolds number form of the standard $k$ $\varepsilon$ equation. The boundary conditions were specified by fixing flow conditions at the inflow, except for pressure, and specifying the back pressure at the outflow boundary. The pressure at the inflow is not specified but adjusts itself to sustain the prescribed mass flow. The simulation results are summarized in Figure 3, which depicts

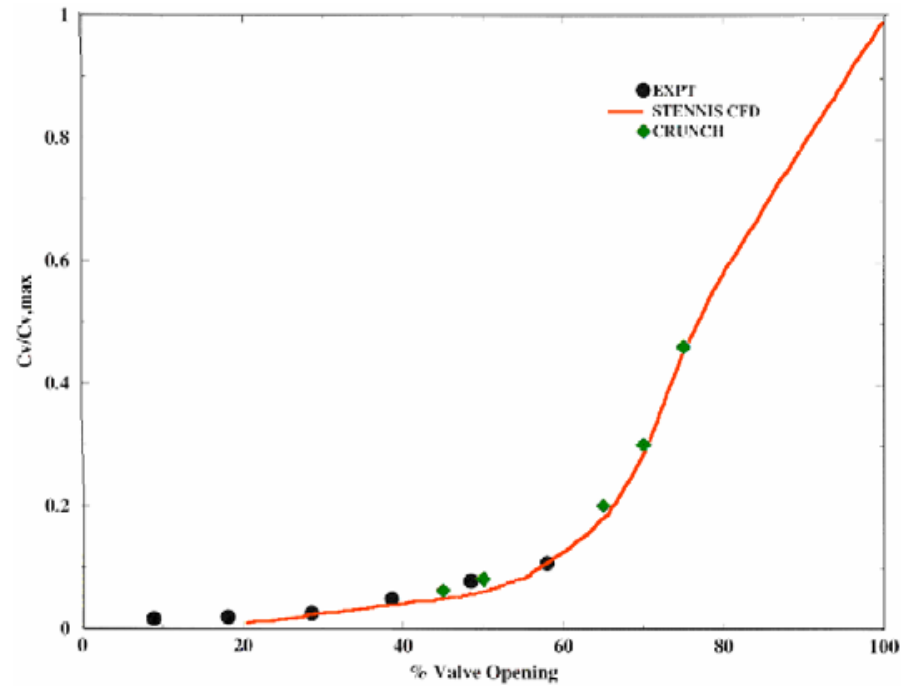

Figure 3. Comparison of the valve flow coefficients with experimental data and Stennis computations. a comparison of the valve flow coefficient as a function of valve position with the experimental observations and calculations performed at Stennis Space Center. The valve flow coefficient, $\mathrm{C}_{\mathrm{v}}$, is defined as the ratio of the integrated mass flow rate $(\mathrm{lbm} / \mathrm{s})$ at the inlet to the square root of pressure differential (psia) required to drive the flow through the control valve. The CRUNCH CFD $^{\circledR}$ simulations show a monotonically increasing valve flow coefficient curve with valve opening, consistent with both the experimental observations and the Stennis calculations. Furthermore, the results are in excellent agreement with experimental measurements and the grid independent values of $\mathrm{C}_{\mathrm{v}}$ computed at Stennis Space Center, both, in predicting the slope of the curve and the transition in slope from the lower valve settings ( $<60 \%$ open) to the higher valve settings ( $>65 \%$ open). The CFD simulations also reveal finer details of the flow physics, identify regions of the valve configurations that are associated with pressure losses, and characterize the mechanics of flow control in the valve system. Further details of the solution are plotted in Figure 4 for a sample plug setting. Figure 4a shows the axial velocity distribution for a plug setting of $75 \%$ open. The flow accelerates as it negotiates the seat region and subsequently turns along the nose of the plug towards the center of the duct. The sharp turning of the flow leads to the formation of large zones of flow separation at the outer walls downstream of the seat region. Stable recirculation patterns are formed in the hollow cavity of the plug for all valve settings. Figure $4 \mathrm{~b}$ shows the turbulent viscosity distribution for the same plug setting, indicative of vorticity and diffusion of the velocity core in the duct downstream of the seat/plug region.

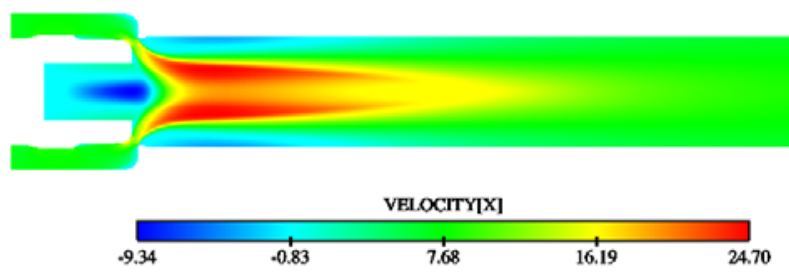

(a)

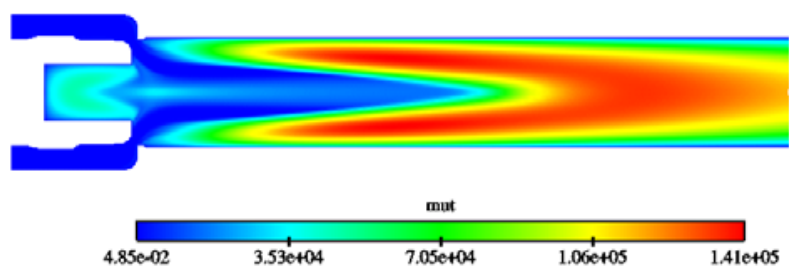

(b)

Figure 4. Plots of (a) axial velocity and (b) turbulent viscosity for the valve at $75 \%$ open. 


\section{Pressure Regulator Valve}

CFD simulations utilizing CRUNCH CFD ${ }^{\circledR}$ were performed for the much more complicated geometry of the gas pressure regulator valve shown in Figure 1. The structural complexity of the geometry and consequently the complex flow path associated with the pressure regulator valve made it particularly suitable for the multi-element framework incorporated in CRUNCH CFD ${ }^{\circledR}$. The multiple corners, disparate shapes, and tight clearances that comprise the flowpath of the valve result in flowfield regions having a variety of length and time scales, as well as diverse flow regimes from low Mach number flow sections to supersonic flow in the seat region. Moreover, the multiple corners and edges have secondary flow structures and transient phenomena such as shedding vortices. Associated with these unsteady phenomena is a dominant chattering-like behavior that has been observed when the valve is operational. Both steady and unsteady simulations were performed with the aim of investigating the processes that occur within the valve that lead to this flow-induced unsteadiness. A description of the methods used to construct the grid and details of the simulations follows.

\section{A. Multi-Element Unstructured Grid Approach}

A multi-element grid comprising of tetrahedral, prismatic, hexahedral and pyramidal cells was constructed for the pressure regulator valve geometry. Traditional structured grids are very difficult to generate for such a complex geometry. For the case of a purely hexahedral grid, the complexity in the geometry forces an awkward block topology with highly skewed and irregular cells. For a contiguous multi-block structured grid, severe constraints on grid resolution are placed on critical regions of the flow domain such as the seat region of the valve where the flow sharply accelerates. Conversely, a grid comprising of only tetrahedral and prism elements is cumbersome to build because of difficulties with prism extrusion in the concave corners and tight clearances associated with the geometry. These shortcomings are overcome in the multi-element approach wherein high quality grids are efficiently generated in all regions of the valve/body geometry. The multi-element unstructured grid was constructed using Gridgen ${ }^{10}$, which allows the flexibility and control of grid resolution needed to rapidly generate a high quality mesh in all the various disparate regions of the valve, and then join them together in a modular fashion.

Figure 5 depicts a planar cross-section of the multielement grid that was generated for the pressure regulator valve simulations. The figure illustrates the various regions in which each cell type was used. In the axisymmetric portions of the geometry, namely the seat region and the areas around the poppet and center of the valve body, hexahedral blocks were created by rotationally extruding quadrilateral surface grids around the valve body axis. The high aspect ratio

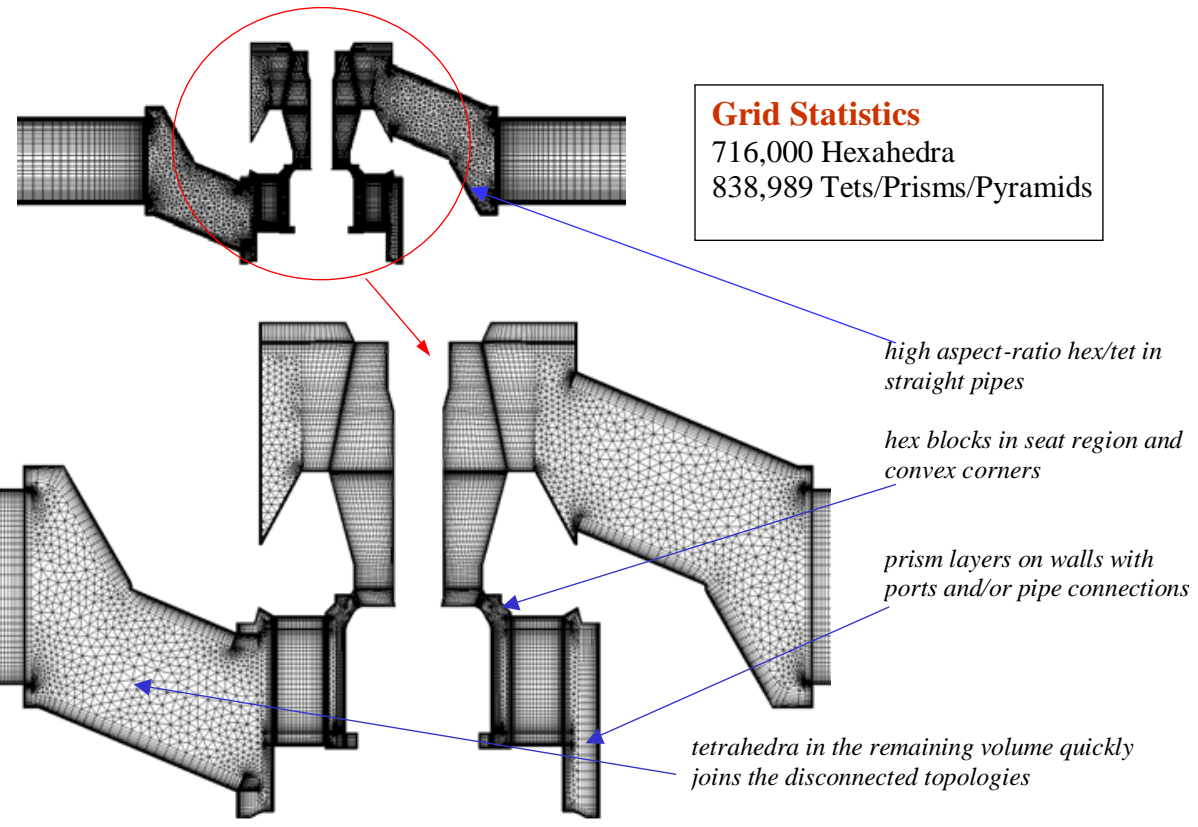

Figure 5. Symmetry plane of the grid illustrating the multi-element mesh topology.

hexahedral cells are ideally suited to resolve the high gradients in the valve seat region and enable boundary layer clustering on the enclosed walls that exist on all sides of the domain. The remaining regions of the valve body and inlet/outlet junctions comprised of non-axisymmetric regions with complex pipe junctions, ports, and acute corners. Prism layers were extruded from triangulated surface grids in this region to resolve the boundary layers associated with these complex surfaces. Straight sections of the geometry such as the inlet pipe, the discharge duct, and the four ports channeling the flow into the valve body were meshed with high aspect ratio hexahedral cells along the walls and a core of prisms through the center. This topology eliminated the "hinge points" that are necessary when an O-H structured 
topology is used for a cylindrical geometry. The remaining portions of the geometry such as the junctions between the inlet/discharge straight portions and the valve housing were filled with tetrahedral cells that acted as buffer zones connecting the disparate topologies in different regions of the valve. The grid was thus created in a modular fashion, allowing appropriate topologies to be applied to each particular region, and then joined together with tetrahedral blocks to close the grid. The resulting mesh consists of 716,000 hexahedral cells with a combination of 838,989 tetrahedral, prismatic and pyramidal cells. Half-plane symmetry was assumed for the simulations.

\section{B. Simulation Results}

Both steady and unsteady simulations were performed with the multi-element grid. A compressible, ideal gas flow was assumed for both simulations. Inflow boundary conditions based on an inlet total pressure of $4400 \mathrm{psi}$ and a total temperature of 540 degrees $\mathrm{R}$ were specified at the inlet plane to the feed duct. At the outflow plane of the discharge duct a back pressure of 800 psi was maintained. The steady simulations revealed a rich variety of flow phenomena over a multitude of length scales, as illustrated in Figure 6. This included a supersonic jet-like penetration of the flow through the seat region, secondary flow patterns in the feed channels and corner regions of the valve housing, a large re-circulation in the upper portion of the valve body, and significant flow expansion to supersonic velocities in the discharge duct. The general structure of the flowfield through the valve is shown in Figure 6a, which shows streamlines colored with Mach number entering the domain from the left side of the figure. In the figure, the solution was mirrored about the symmetry plane in order to visualize the full valve domain. The streamlines show some of the secondary flow structures present in the feed channels and the large recirculation that develops in the top of the valve body as the flow negotiates the transition from the valve assembly to the discharge pipe. Figure $6 \mathrm{~b}$ shows the Mach number distribution along the plane of symmetry. It can be seen that the flow mildly accelerates as it transitions from the intake pipe to the feed ports leading into the valve housing. The flow sharply accelerates through the narrow clearance of the valve seat region. The valve seat acts as a throat, accelerating the flow to supersonic velocities. The flow emerges from the valve seat region like a jet into the upper housing where it turns and transitions into the discharge pipe. The impingement of the jet on the upper surface of the valve body causes the recirculation region visible in figure 6b. The Mach number distribution reveals flow expansion to supersonic velocities into the discharge pipe as the flow turns the corners within the pipe junction region. Figure 6c shows the pressure distribution on the poppet and shaft surfaces. The figure reveals the large pressure drop that occurs across the valve seat at this particular plug setting and a spot of high pressure on the shaft just downstream of the throat, resulting from a stagnation point within the interaction of the multiple flow streams entering the valve from the feed ports.

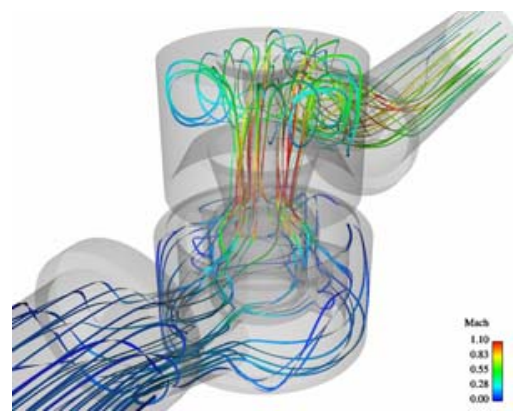

(a)

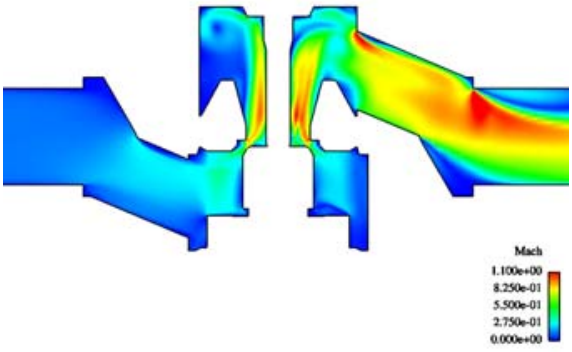

(b)

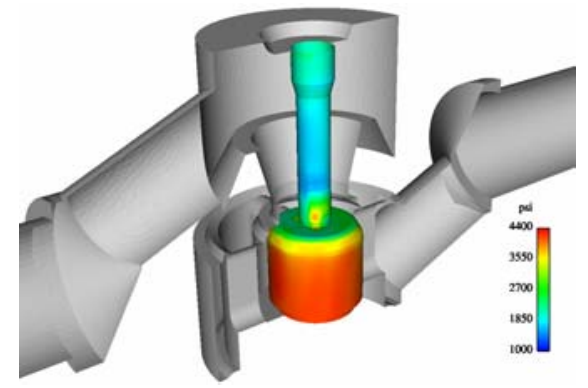

(c)

Figure 6. Plots of (a) streamlines through the valve colored with Mach number illustrating the large recirculation region, $(b)$ Mach number contours on the symmetry plane, and $(c)$ pressure contours on the poppet/shaft surfaces.

The unsteady modeling effort was geared towards understanding an observed "chatter" of the poppet-shaft assembly during operation of the valve. A transient analysis with the same fixed plug setting was performed with a time step of $3 \times 10^{-7}$ seconds, allowing frequencies of up to several hundred $\mathrm{KHz}$ to be adequately resolved. Starting from the steady state solution, the simulation was run for 12,000 iterations for a simulated time of 3.6 milliseconds and captured a dominant periodic oscillation in the flow with a frequency close to $4 \mathrm{KHz}$. For the time period of the simulation, about 14 cycles of data were collected. Figure 7 shows the progression of the simulation at intervals of $\mathrm{T} / 8$, where the characteristic time period roughly corresponds to one cycle of the oscillation. The sequence of Mach number distribution plots depicts a periodic breakup of the jet-like structure emanating from the narrow clearance around the valve seat/throat. The pulsing of the flow here is most pronounced on the inlet duct side of the valve body. This flow then periodically discharges tangentially through the exhaust duct. Flow in the discharge duct 
periodically transitions to supersonic flow as a result of the exhaust from the upper portion of the valve body. The sequence of cross-sectional plots in Figure 8 clearly shows the fluctuation in the tangential discharge towards the side of the upper portion of the valve body facing the exhaust duct. Furthermore, this tangential discharge has a similar periodic structure to that of the jet-like structure discussed above that represents the dominant axial mode instability in the valve. Through these flow visualizations of the transient solution, the source of the instability was identified as an axial mode, which manifested itself as a periodic pulsation of a jet-like structure through the throat coupled with tangential modes in the discharge portion of the flow.
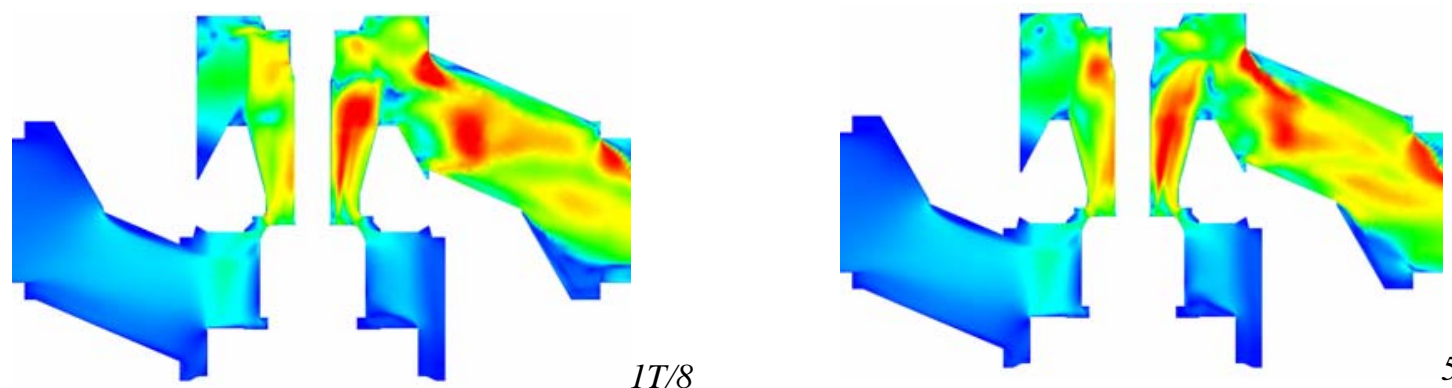

$1 T / 8$
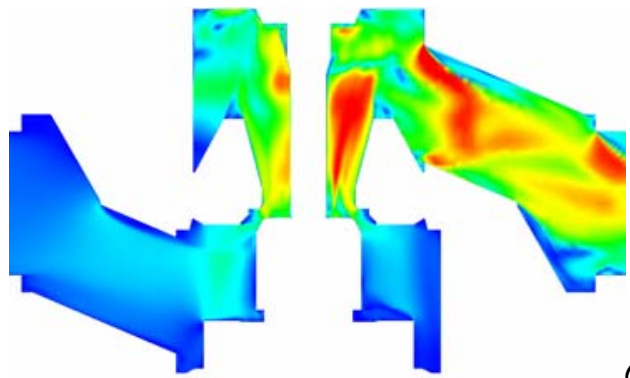

$5 T / 8$
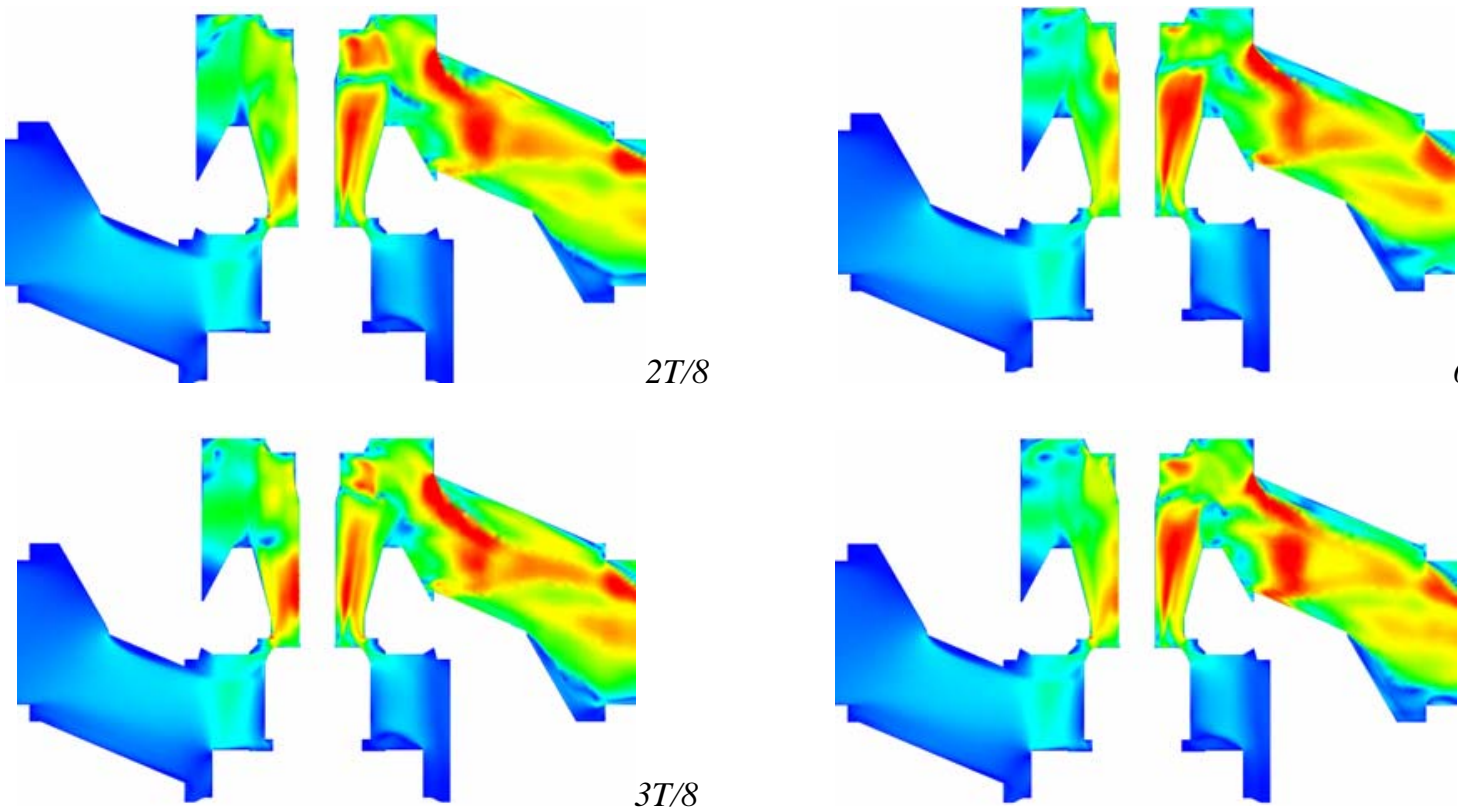

$6 T / 8$
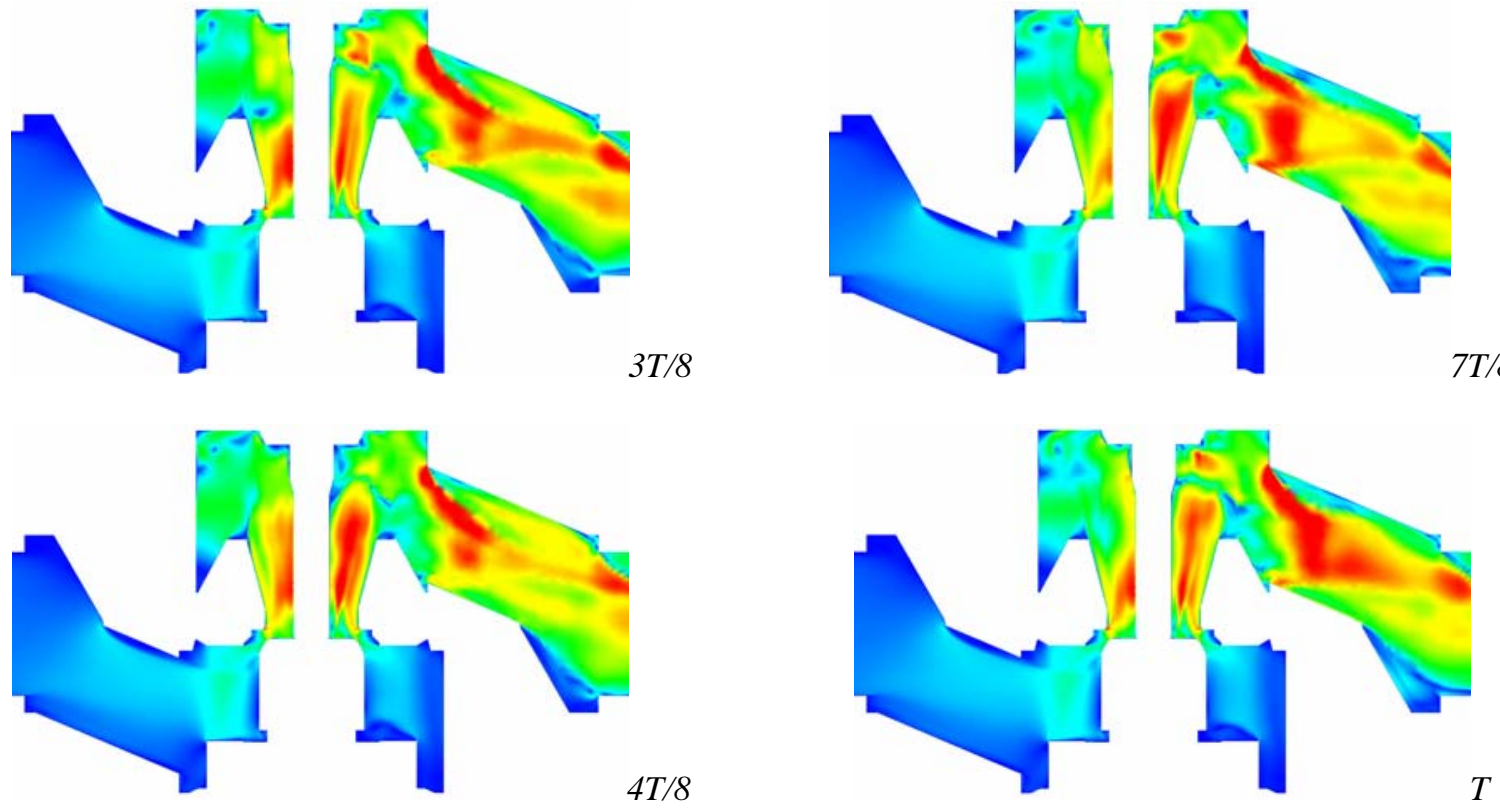

Figure 7. Sequence of Mach Number Distribution in the Pressure Regulator Valve as the Unsteady Simulation Evolves. 

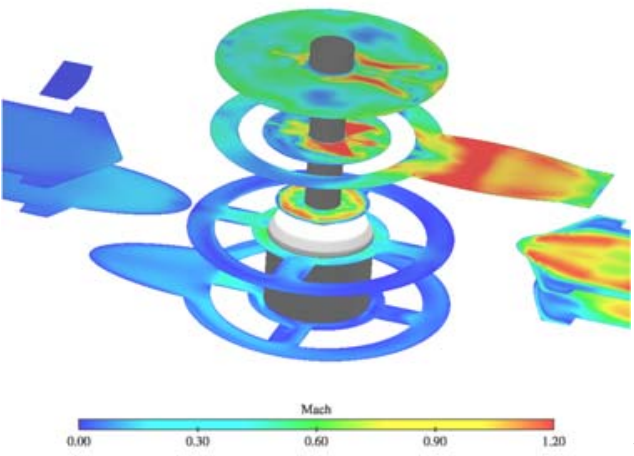

$1 \mathrm{~T} / 8$
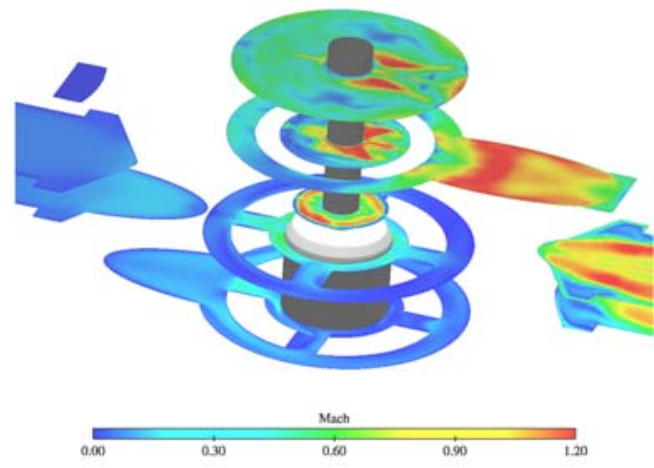

$2 \mathrm{~T} / 8$
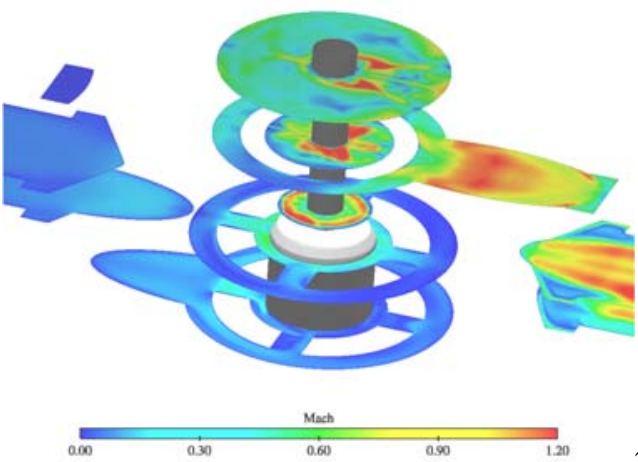

$3 \mathrm{~T} / 8$
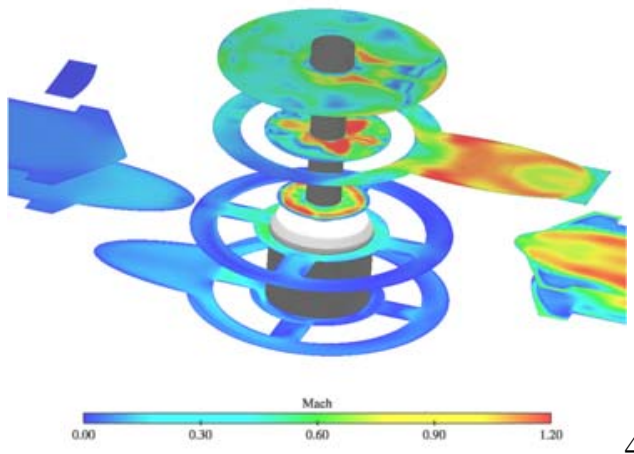
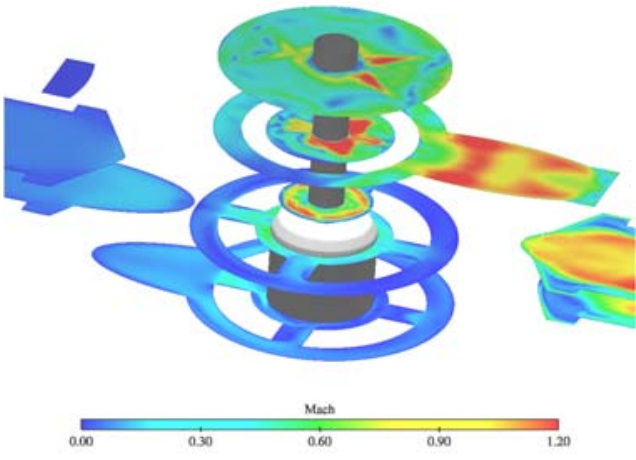

$5 \mathrm{~T} / 8$
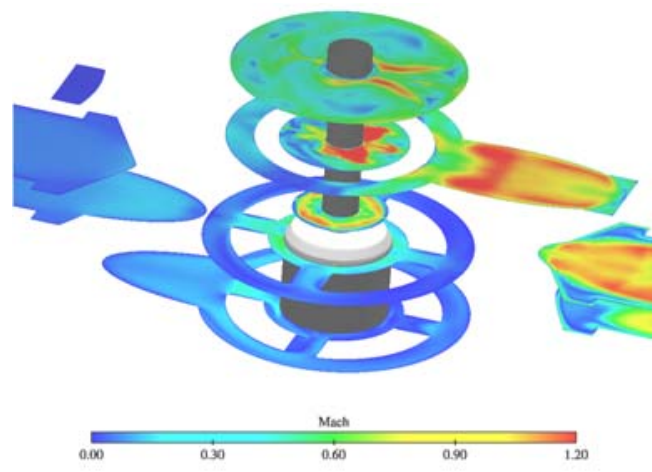

$6 \mathrm{~T} / 8$
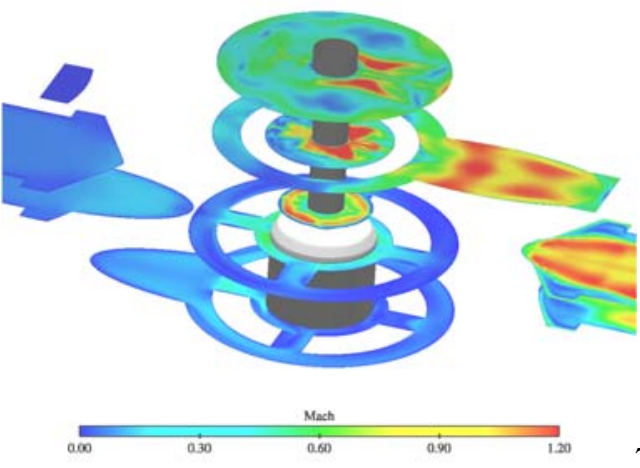

$7 \mathrm{~T} / 8$
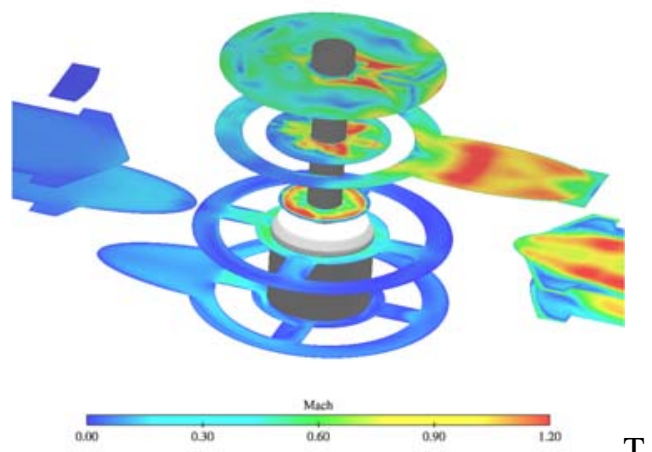

$\mathrm{T}$

Figure 8. Sequence of Planar Cross Sections of Mach Number Distribution Showing Tangential Discharge in Upper Housing of Pressure Regulator Valve. 
Pressure histories were recorded at seven different points on the poppet close to the throat for the length of the simulation. The probe points were mostly distributed along the base of the throat on the poppet. Three pairs of points were located on the base (on the periphery and midway on radial lines running from the shaft to the outer edges) aligned with the direction of the feed channels in the lower portion of the valve body. One of the points was placed on the shaft at the location where the steady state simulation indicated a localized region of high pressure. Figure 9a illustrates the locations of the seven probe points along the poppet surface. All seven probes show an almost identical periodic variation in pressure, as shown in Figure 9b. The variations are strongest at the three points on the periphery of the base region $(1,2$ and 3$)$ that also coincides with the throat region of the valve. A Fourier analysis of the pressure history reveals the frequency content of the instability modes, as shown in Figure 9c. This analysis indicates that most of the energy is associated with a fundamental mode corresponding to a frequency of $4043 \mathrm{~Hz}$. Significant energy is also contained in the first harmonic (around $8 \mathrm{KHz}$ ). A closer look at Figure 9c shows that the simulation resolves an additional three or four harmonics. Furthermore, the Fourier analysis reveals an active low frequency mode of approximately $250 \mathrm{~Hz}$. This mode could be structurally significant in that it could excite poppet vibration modes leading to significant noise and couple with structural modes leading to potential structural failure.

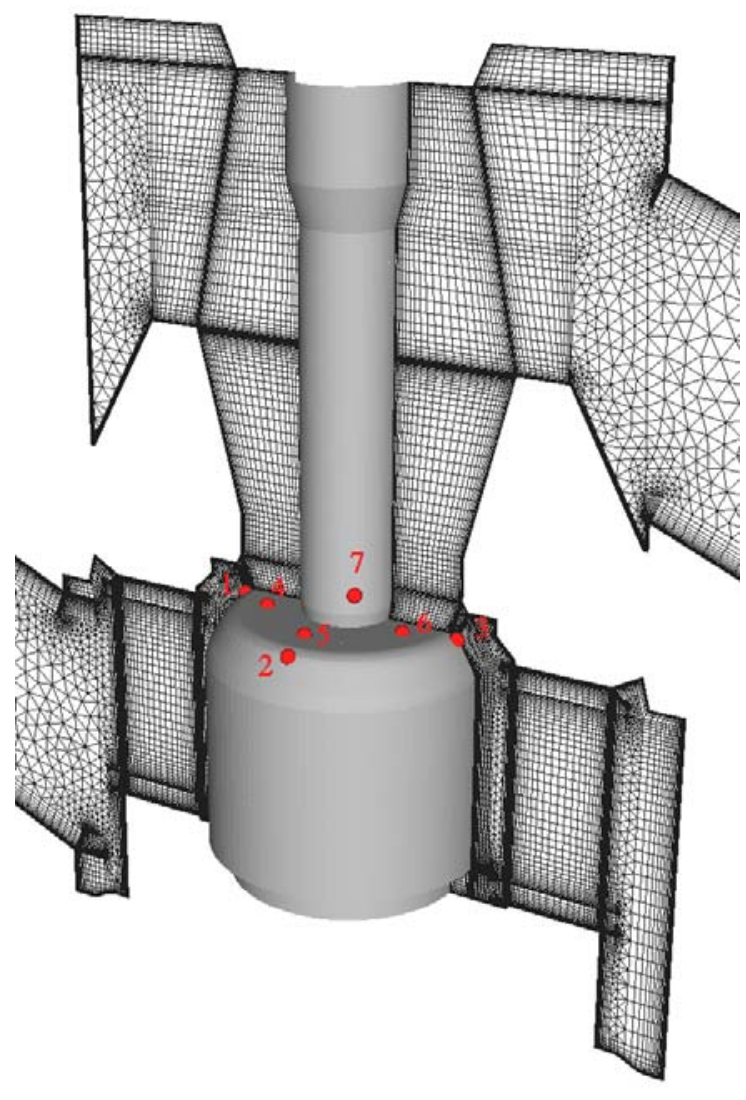

(a)

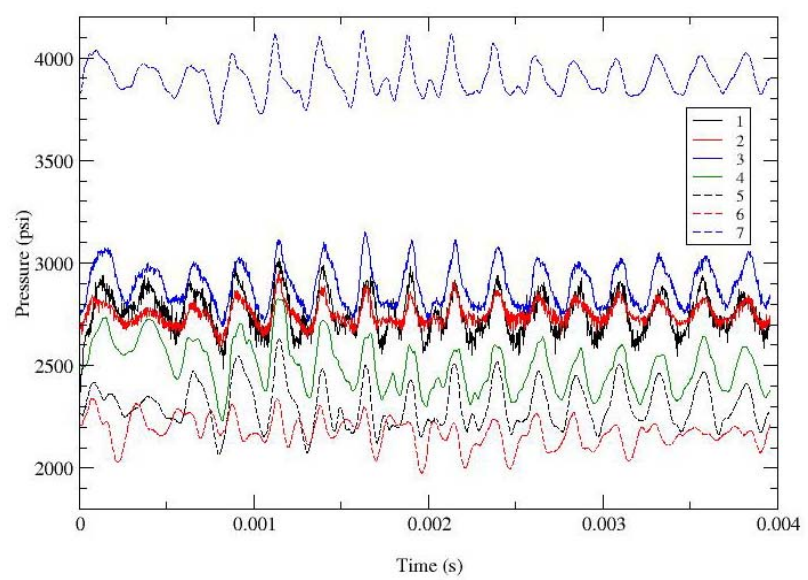

(b)

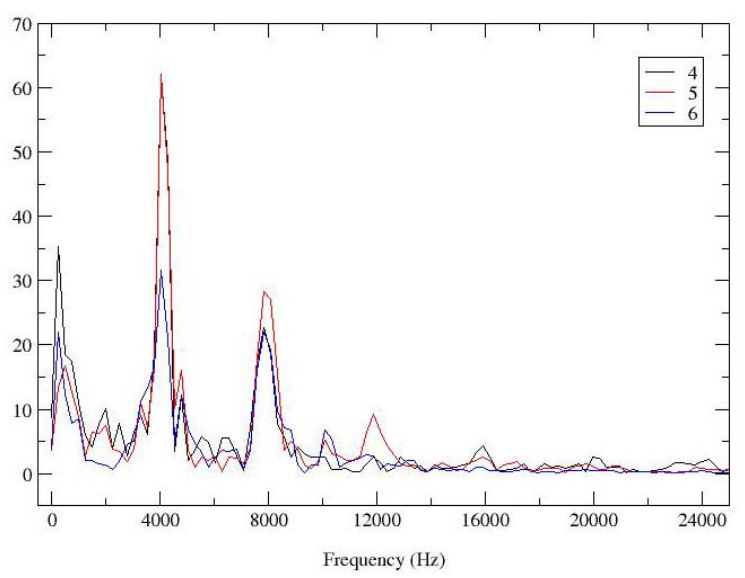

(c)

Figure 9. Plots of the transient solution showing (a) location of probe points on the poppet, $(b)$ pressure history for the seven points, and $(c)$ frequency spectrum indicating the dominant instability modes.

\section{Conclusion}

Simulations were presented illustrating the application of the multi-element unstructured framework contained in the CRUNCH CFD ${ }^{\circledR}$ code to complex valve systems of the type used in feed systems for liquid rocket engine test installations. The ability of the multi-element approach to efficiently generate high quality grids for the complex geometries typical of valve systems was demonstrated. Simulations were performed for a cryogenic control valve in 
order to validate the simulation methodology with both experimental data and simulations performed at Stennis Space Center. Detailed steady and unsteady analyses were presented for a gas pressure regulator valve found in the high pressure feed systems of the rocket test facilities at NASA Stennis Space Center. The unsteady simulation revealed a strong periodic instability with a frequency close to $4 \mathrm{KHz}$. Flow visualization of the transient solution indicates that the source of this instability is an axial mode coupled with fluctuations related to periodic shedding in the discharge portion of the valve body. The results help understand the processes that occur in the valve flowpath leading to the high frequency chattering observed in the operational valve at high flow conditions.

\section{Acknowledgments}

We acknowledge funding for this work through a NASA Phase I SBIR, Contract Number NAS13-03009 funded by NASA Stennis Flight Center. Dr. Peter Sulyma was the technical monitor and the technical inputs by Dr. Russell Daines and Mr. Jody Woods are also greatly acknowledged.

\section{References}

1 Daines, R.L., Woods, J.L., and Sulyma, P.R., “Progress in Valve Modeling at Stennis Space Center,"Penn State Propulsion Engineering Research Center Fourteenth Annual Symposium, State College, PA, December 2002.

2 Ahuja, V., Hosangadi, A. and Shipman, J., “Multi-Element Unstructured Analyses of Complex Valve Systems,” 52nd JPM/1st LPS Meeting, Las Vegas, NV, May 10-14, 2004.

3 Ahuja, V., Hosangadi, A. and Shipman, J., “Computational Analyses Of Cavitating Control Elements In Cryogenic Environments,” Paper No. HT-FED2004-56377, The 2004 Joint ASME-JSME Fluids Engineering Summer Conference, Charlotte, N.C., July 11-15, 2004

4 Ahuja, V., Shipman, J.D., Arunajatesan, S., and Hosangadi, A., "Multi-Element Unstructured Methodology for Analysis of Turbomachinery Systems" Journal of Propulsion and Power, Vol. 19, No. 5, pp. 945-952, Sept-Oct 2003.

5 Hosangadi, A., Cavallo, P.A., Arunajatesan, S., Ungewitter, R. and Lee, R.A., “Aero-Propulsive Jet Interaction Simulations Using Hybrid Unstructured Meshes,” AIAA Paper-99-2219, 35th AIAA/ASME/SAE/ASEE JPC, Los Angeles, CA, June 20-24, 1999.

6 Barth, T.J., “A 3-D Upwind Euler Solver for Unstructured Meshes,” Paper No. AIAA-91-1548.

7 Hosangadi, A., Lee, R.A., York, B.J., Sinha, N., and Dash, S.M., "Upwind Unstructured Scheme for Three-Dimensional Combusting Flows," Journal of Propulsion and Power, Vol. 12, No. 3, pp. 494-503, May-June 1996.

8 Cavallo, P.A., Sinha, N., and Feldman, G.M., "Parallel Unstructured Mesh Adaptation for Transient Moving Body and Aeropropulsive Applications”, AIAA Paper 2004-1057, 42nd Aerospace Sciences Meeting and Exhibit, Reno, NV, January 5-8, 2004.

9 Daines, R.L., Woods, J.L., and Sulyma, P.R., “Computation Analysis of Cryogenic Flow Through a Control Valve,” FEDSM2003-45120, 4th ASME-JSME Joint Fluids Engineering Conference, Honolulu, Hawaii, July 2003.

${ }^{10}$ Gridgen, Grid Generation Software Package, Ver. 15, Pointwise, Inc., Ft. Worth, TX, 2004. 